\section{Whole Genome Sequence and Gene Annotation Resource for Didymella bellidis Associated with Tea Leaf Spot}

\author{
Xue Wang, ${ }^{1}$ Xian Wu, ${ }^{1}$ Silong Jiang, ${ }^{2}$ Qiaoxiu Yin, ${ }^{1}$ Dongxue Li, ${ }^{1}$ Yong Wang, ${ }^{2}$ Delu Wang, ${ }^{3, \dagger}$ \\ and Zhuo Chen ${ }^{1, t}$ \\ ${ }^{1}$ Key Laboratory of Green Pesticide and Agricultural Bioengineering, Ministry of Education, Guizhou \\ University, Guiyang, Guizhou 550025, China \\ ${ }^{2}$ Agricultural College, Guizhou University, Guiyang, Guizhou 550025, China \\ ${ }^{3}$ College of Forestry, Guizhou University, Guiyang, Guizhou 550025, China
}

\begin{abstract}
Didymella bellidis is a phytopathogenic fungus that causes leaf spot on tea plants (Camellia sinensis), which negatively affects the productivity and quality of tea leaves in Guizhou Province, China. D. bellidis isolate GZYQYQX2B was sequenced using Pacific Biosciences and Illumina technologies, and assembled into a whole genome of $35.5 \mathrm{Mbp}$. Transcripts of $D$. bellidis isolate GZYQYQX2B were predicted from the assembled genome and were further validated by RNA sequence data. In total, 10,731 genes were predicted by integrating three approaches, namely $a b$ initio and homology-based gene prediction, as well as transcriptomics data. The whole-genome sequence of $D$. bellidis will provide a resource for future research on trait-specific genes of the pathogen and host-pathogen interactions.
\end{abstract}

In recent years, tea (Camellia sinensis [L.] Kuntze) has been widely cultivated in Guizhou Province, China, where the cultivated area has reached 350,000 ha, making it the major teagrowing province in China (Bao et al. 2019; Li et al. 2019). Tea leaf spot, caused by Didymella bellidis (Neerg.) Chen \& Cai, occurs widely throughout the tea plantations of Guizhou Province, seriously affecting both the productivity and quality of tea leaves (Wang et al. 2020).

$D$. bellidis belongs to the family Didymellaceae, which is the largest family in the order Pleosporales (phylum Ascomycota, subdivision Pezizomycotina, class Dothideomycetes) (Crous et al. 2004). Based on multilocus sequence analysis, the Didymellaceae is composed of 19 monophyletic generic clades (Chen et al. 2017). Didymella is one of the most speciesrich genera in the Didymellaceae (Chen et al. 2017). D. bellidis is a species that is regarded as being closely related to $D$. segeticola, based on multilocus analysis of the partial sequences of the large subunit of rDNA (LSU), the internal transcribed spacer (ITS) of rDNA, the second largest subunit of RNA polymerase II (RPB2), and $\beta$-tubulin (tub2), as well morphological traits common to the two species, such as pycnidia, conidiogenous cells, and conidia (Chen et al. 2015, 2017; Wang et al. 2020). D. bellidis has been reported to cause leaf spot on Bellis perennis (Chen et al. 2015), Eleocharis dulcis (Lv et al. 2011), Angelica dahurica (Xu et al. 2016), Chrysanthemum morifolium (Liu et al. 2019), and Camellia sinensis (Wang et al. 2020). D. segeticola and Epicoccum sorghinum can also cause tea leaf spot in Guizhou Province (Bao et al. 2019; Ren et al. 2019b). D. bellidis, D. segeticola, and E. sorghinum cause similar symptoms on tea leaf, although a pathogenicity test under laboratory conditions indicated that the virulence of $E$. sorghinum was greater than that of the other pathogens, with $D$. segeticola being the weakest of the three species in terms of lesion size and lesion

\footnotetext{
†Corresponding authors: D. L. Wang; dlwang@gzu.edu.cn, and Z. Chen; gychenzhuo@aliyun.com
}

*The $e$-Xtra logo stands for "electronic extra" and indicates that one supplementary table is published online.

The author(s) declare no conflict of interest.

Accepted for publication 24 September 2020

(C) 2021 The American Phytopathological Society

1168 Plant Disease /Vol. 105 No. 4

$e-$ tra $^{*}$

\section{Funding}

The project was supported by National Key Research Development Program of China (2017YFD0200308) and its post-subsidy project (2018-5262), the National Natural Science Foundation of China (no. 21977023).

\section{Keywords}

genome, fungus-plant interactions, leaf spot 
Table 1. Sequence data of the whole genome and transcriptome of Didymella bellidis isolate GZYQYQX2B

\begin{tabular}{lc} 
Parameters & Results \\
Contig number & 85 \\
Contig length (bp) & $35,580,733$ \\
GC content (\%) & 51.40 \\
Contig MIN (bp) & 1,122 \\
Contig N50 (bp) & $1,967,293$ \\
Contig N90 (bp) & 400,412 \\
Contig MAX (bp) & $3,873,157$ \\
Number of predicted genes & 10,731 \\
Number of transcripts & 10,731 \\
Percentage completeness & $98.6 \%$ \\
\hline
\end{tabular}

formation rate (Bao et al. 2019; Ren et al. 2019a; Wang et al. 2020). The genome of $D$. segeticola was sequenced by our research group (BioProject accession: PRJNA516041, BioSample: SAMN10768991, SRA: SRR8492863) (Ren et al. 2019b).

The $D$. bellidis isolate GZYQYQX2B was obtained by single spore isolation from leaf spot-infected tea leaves in Yuqing County, Guizhou Province (Wang et al. 2020). This isolate was grown in sterile potato dextrose broth (PDB) at $28^{\circ} \mathrm{C}$ and $180 \mathrm{rpm}$ in the shaker incubator for 10 days before mycelia were harvested and ground with liquid nitrogen. Genomic DNA was then isolated using a fungal DNA miniprep kit (E.Z.N.A., Omega Bio-tek, Inc., Norcross, GA, U.S.A). Since the size of the main band of genomic DNA of the fungus is $15 \mathrm{kbp}$ using pulsed-field gel electrophoresis, fragments of approximately $15 \mathrm{kbp}$ were prepared using the G-tube fragmentation to construct the PacBio RS II library (Covaris M220 Focusedultrasonicator; Thermo Fisher Scientific, Inc. Woburn, MA, U.S.A.). The size of the PacBio RS II library was assessed using an Agilent 2100 bioanalyzer (Agilent Technologies, Inc., Santa Clara, CA, U.S.A.). After short polymerase reads, low-quality sequences and adapter sequences with self-linkers were removed, polymerase reads generated by a PacBio Sequel system (LC-bio Technologies Co., Ltd., Hangzhou, China) formed subreads using SMRT Link (version 8.0) (https://www.pacb.com/support/software-downloads/). The mean read length was $9.76 \mathrm{kbp}$, with an N50 of $12.93 \mathrm{kbp}$. The number of reads longer than $5 \mathrm{Kbp}$ was $1,372,236(73.59 \%$ of the total reads), with the number of bases in reads longer than $5 \mathrm{Kbp}$ being $17,037,452,578$ (93.62\% of the total bases). Sequencing depth was estimated to be $\sim 535 \times$ based on genome size. Raw reads with 16,081,316 were generated by the lllumina HiSeq PE technology (Lc-bio Technologies Co., Ltd., Hangzhou, China). The raw reads were trimmed, corrected, and filtered using a Python 3 script, and 16,004,977 filtered reads were used in subsequent analyses. The percentage GC and Q30 (the ratio of bases with quality values longer than 30 ) were 50.44:50.60 and 95.89:90.64, respectively. Transcriptome data from $D$. bellidis isolate GZYQYQX2B were used to assist in gene prediction. Transcripts were generated from $D$. bellidis isolate GZYQYQX2B cultured on potato dextrose broth (PDB, Beijing Solarbio Science \& Technology Co., Ltd., Beijing, China) under conditions of darkness, $25^{\circ} \mathrm{C}$, and $180 \mathrm{rpm}$ in the shaker incubator for three periods of 70, 105, and $120 \mathrm{~h}$. RNASeq libraries were prepared using an Illumina TruSeq Stranded mRNA Library Prep Kit (Illumina, San Diego, CA, U.S.A.), with an average insert size of $300 \mathrm{bp}( \pm 50 \mathrm{bp})$ for the paired-end libraries. Paired-end sequencing on an Illumina Novaseq 6000 sequencer (Illumina, San Diego, CA, U.S.A) was conducted following the manufacturer's recommended protocol. Cutadapt (Martin 2011) and in-house Perl scripts were used to remove the reads that contained adapter contamination, low-quality reads, and undetermined bases. Sequence quality was assessed using FastQC (http://www.bioinformatics.babraham.ac.uk/projects/ fastqc/), including the Q20 and Q30 indices (the ratio of bases with quality values longer than 20 or 30) and the GC-content of the clean data. De novo assembly of the transcriptome was performed with Trinity 2.4 .0 (https://github.com/trinityrnaseq/trinityrnaseq/releases/tag/Trinity-v2.4.0) (Grabherr et al. 2011). PacBio subreads were assembled using CANU (version 1.6) (https://github.com/marbl/canu/releases). After PacBio subreads were corrected, the data were aligned with the Illumina reads and the bases were further corrected using Pilon (version 1.16) (https://github.com/broadinstitute/pilon/releases). Long and short reads from PacBio (Pacific Biosciences, Inc., Menlo Park, CA, U.S.A) and Illumina technologies (Illumina Inc., San Diego, CA, U.S.A) were used to generate sequences, and then to form hybrid 
assemblies. Completeness of the genome assemblies was assessed using Benchmarking Universal Single-Copy Orthologs (BUSCO) (version 3.0.2) (https://busco.ezlab.org) (Simão et al. 2015). An assessment of genome completeness showed $98.6 \%$ completeness with $97.9 \%$ complete single-copy BUSCOs, $0.7 \%$ complete duplicated BUSCOs, $0.0 \%$ fragmented BUSCOs, and $1.4 \%$ missing BUSCOs from a total of 290 BUSCO groups searched. A summary of the genome assemblies and gene prediction is shown in Table 1. The assembly size of $D$. bellidis was similar to the approximate genome size of $D$. segeticola ( $\sim 33.4 \mathrm{Mbp}$; Ren et al. 2019b). Gene prediction, using ab initio (Augustus software) (http://augustus.gobics.de/binaries), homology, and transcriptome data for the assembled genome of $D$. bellidis isolate GZYQYQX2B identified 10,731 protein-coding genes (number of exons = 33,049, number of introns $=22,318$ ). The functional annotation of the genes was carried out using the Kyoto Encyclopedia of Genes and Genomes (KEGG) (https://www.genome.jp/ kegg/) (Moriya et al. 2007), Swiss-Prot (ftp://ttp.ebi.ac.uk/pub/databases/uniprot/knowledgebase/uniprot_sprot.fasta.gz) (Bairoch and Apweiler 2000), eggNOG (version 4.5) (HuertaCepas et al. 2016), Carbohydrate-Active Enzymes (CAZYme) (http://www.cazy.org) (Yin et al. 2012), the Pathogen Host Interactions database (PHI) (http://www.phi-base.org) (Winnenburg et al. 2006) and transport proteins (Transporter Classification Database, TCDB) (http://www.tcdb.org/) by BLAST software. The results of the functional annotation indicated that 3,511 (32.72\%), 6,728 (62.7\%), 5,933 (55.29\%), 9,792 (91.25\%), and 7,727 (72.01\%) of the genes were annotated in the databases of KEGG, GO, Swiss-Prot, eggnog, or Pfam, as well as $703(6.55 \%), 3,933(36.65 \%)$, and $1,688(15.7 \%)$ in the proprietary databases of CAZYme, PHI, and TCDB, respectively.

The genome sequence of $D$. bellidis will provide an important resource for researchers studying pathogen detection, epidemiology, population genetics, and disease resistance. Comparative genomics with other pathogens of tea, namely $D$. segeticola and $E$. sorghinum, will help us better understand the pathogenic mechanism and epidemiology of tea leaf spot. The genome sequence has been deposited in GenBank under BioProject (PRJNA601275), BioSample (SAMN13864724), and SRA (SRR10896320). The annotated transcripts have been deposited at GenBank under BioProject ID PRJNA604665. In addition, the complete transcript annotations, along with the transcript IDs and the relevant $\mathrm{NCBI}$ accession numbers, are presented in Supplementary Table S1. The $D$. bellidis isolate GZYQYQX2B has been deposited in China General Microbiological Culture Collection Center, with the preservation number of fungus as CGMCC3.20149.

\section{Literature Cited}

Bairoch, A., and Apweiler, R. 2000. The SWISS-PROT protein sequence database and its supplement TrEMBL in 2000. Nucleic Acids Res. 28:45-48.

Bao, X.-T., Dharmasena, D. S. P., Li, D.-X., Wang, X., Jiang, S.-L., Ren, Y.-F., Wang, D.-L., Song, B.-A., and Chen, Z. 2019. First report of Epicoccum sorghinum causing leaf spot on tea in China. Plant Dis. 103:3282.

Chen, Q., Hou, L.-W., Duan, W.-J., Crous, P. W., and Cai, L. 2017. Didymellaceae revisited. Stud. Mycol. 87:105-159.

Chen, Q., Jiang, J.-R., Zhang, G.-Z., Cai, L., and Crous, P. W. 2015. Resolving the Phoma enigma. Stud. Mycol. 82:137-217.

Crous, P. W., Gams, W., Stalpers, J. A., Robert, V., and Stegehuis, G. 2004. MycoBank: an online initiative to launch mycology into the 21st century. Stud. Mycol. 50:19-22.

Grabherr, M. G., Haas, B. J., Yassour, M., Levin, J. Z., Thompson, D. A., Amit, I., Adiconis, X., Fan, L., Raychowdhury, R., Zeng, Q., Chen, Z., Mauceli, E., Hacohen, N., Gnirke, A., Rhind, N., di Palma, F., Birren, B. W., Nusbaum, C., Lindblad-Toh, K., Friedman, N., and Regev, A. 2011. Full-length transcriptome assembly from RNAseq data without a reference genome. Nat. Biotechnol. 29:644-652.

Huerta-Cepas, J., Szklarczyk, D., Forslund, K., Cook, H., Heller, D., Walter, M. C., Rattei, T., Mende, D. R., Sunagawa, S., Kuhn, M., Jensen, L. J., von Mering, C., and Bork, P. 2016. eggNOG 4.5: A hierarchical orthology framework with improved functional annotations for eukaryotic, prokaryotic and viral sequences. Nucleic Acids Res. 44(D1):):D286-D293.

Li, D.-X., Bao, X.-T., Ren, Y.-F., Song, B.-A., and Chen, Z. 2019. First report of Lasiodiplodia theobromae causing leaf spot on tea plant in Guizhou Province of China. Plant Dis. 103:374

Liu, Y.-H., Zhang, C.-Q., and Dai, D.-J. 2019. First report of leaf black spot on White Chrysanthemum (Chrysanthemum morifolium) caused by Phoma bellidis in China. Plant Dis. 103:2475.
Lv, R., Zheng, L., Zhu, Z., Pan, L., Huang, J., and Hsiang, T. 2011. First report of stem blight of Eleocharis dulcis caused by Phoma bellidis in China. Plant Dis. 95: 1190.

Martin, M. 2011. Cutadapt removes adapter sequences from high-throughput sequencing reads. EMBnet. J. 17:10-12.

Moriya, Y., Itoh, M., Okuda, S., Yoshizawa, A. C., and Kanehisa, M. 2007. KAAS: An automatic genome annotation and pathway reconstruction server. Nucleic Acids Res. 35:W182-W185.

Ren, Y.-F., Bao, X.-T., Li, D.-X., Wang, Y., Wang, D.-L., Song, B.-A., and Chen, Z. 2019a. Identification of the pathogen Lasiodiplodia theobromae causing tea leaf spot. Acta Phytopathologica Sin. 49:857-861.

Ren, Y.-F., Li, D.-X., Zhao, X.-Z., Wang, Y., Bao, X.-T., Wang, X., Wu, X., Wang, D.-L., Song, B.-A., and Chen, Z. 2019b. Whole genome sequences of the tea leaf spot pathogen Didymella segeticola. Phytopathology 109:1676-1678.

Simão, F. A., Waterhouse, R. M., Ioannidis, P., Kriventseva, E. V., and Zdobnov, E. M. 2015. BUSCO: assessing genome assembly and annotation completeness with single-copyorthologs. Bioinformatics 31:3210-3212.

Wang, X., Yin, Q.-X., Jiang, S.-L., Wu, X., Wang, D.-L., Song, B.-A., and Chen, Z. 2020. First report of Didymella bellidis causing tea leaf spot in China. Plant Dis. 104:1254.

Winnenburg, R., Baldwin, T. K., Urban, M., Rawlings, C., Köhler, J., and HammondKosack, K. E. 2006. PHI-base: A new database for pathogen host interactions. Nucleic Acids Res. 34:D459-D464.

Xu, H.J., Cui, J.-C., Zhou, R.-J., Fu, J.-F., and Hao, N. 2016. First report of leaf spot disease in Angelica dahurica caused by Phoma bellidis in China. J. Phytopathol. 164:448-454.

Yin, Y., Mao, X., Yang, J., Chen, X., Mao, F., and Xu, Y. 2012. dbCAN: A web resource for automated carbohydrate-active enzyme annotation. Nucleic Acids Res. 40:W445-W451. 